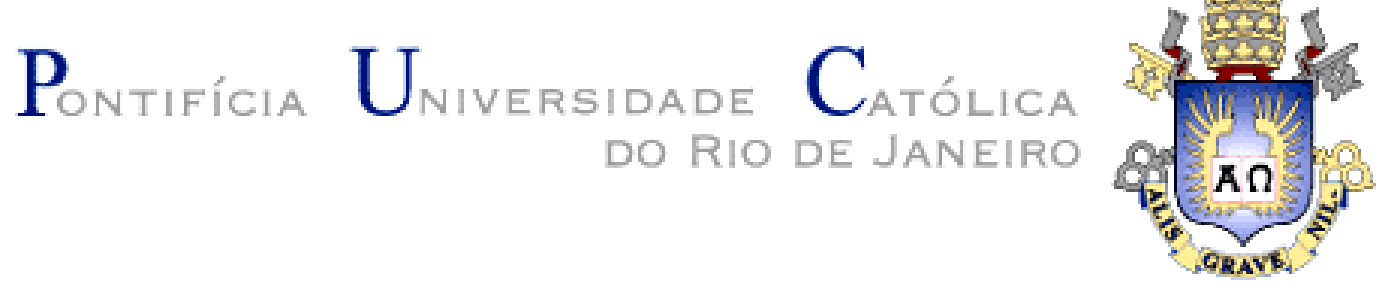

Juan David Velilla Uribe

\title{
Estabilidade de Poços de Petróleo em Meios Fraturados Empregando o Método dos Elementos Discretos
}

Dissertação de Mestrado

Dissertação apresentada como requisito parcial para obtenção do grau de Mestre pelo Programa de Pós-Graduação em Engenharia Civil do Departamento de Engenharia Civil da PUC-Rio.

Orientador: Sérgio Augusto Barreto da Fontoura

Co-orientador: Nelson Inoue

Rio de Janeiro, março de 2013. 


\title{
Juan David Velilla Uribe
}

\section{Estabilidade de Poços de Petróleo em Meios Fraturados Empregando o Método dos Elementos Discretos}

\begin{abstract}
Dissertação apresentada como requisito parcial para obtenção do grau de Mestre pelo Programa de Pós-Graduação em Engenharia Civil do Departamento de Engenharia Civil do Centro Técnico Cientifico da PUC-Rio. Aprovada pela Comissão Examinadora abaixo assinada.
\end{abstract}

Prof. Sérgio Augusto Barreto da Fontoura Orientador Departamento de Engenharia Civil-PUC-RIO

\author{
Dr. Nelson Inoue \\ Co-orientador \\ GTEP/PUC-Rio
}

Prof. Ney Augusto Dumont Departamento de Engenharia Civil-PUC-RIO Dr. Erick Slis Raggio Santos
CENPES/PETROBRAS

Prof. José Eugenio Leal

Coordenador Setorial do Centro Técnico Cientifico- PUC-Rio

Rio de Janeiro, 25 de março de 2013. 
Todos os direitos reservados. É proibida a reprodução total ou parcial do trabalho sem autorização da universidade, do autor e do orientador.

\section{Juan David Velilla Uribe}

Graduou-se em Engenharia de Petróleos na UIS (Universidade Industrial de Santander - Colômbia) em Março de 2010. No mesmo ano trabalho na área de Geomecânica do Petróleo no ICP (Instituto Colombiano do Petróleo). No ano 2011 ingressou ao curso de Mestrado em Engenharia Civil na Pontifícia Universidade Católica do Rio de Janeiro, na área de Geotécnia, desenvolvendo dissertação de mestrado na linha de pesquisa de Geomecânica do Petróleo.

Ficha Catalográfica

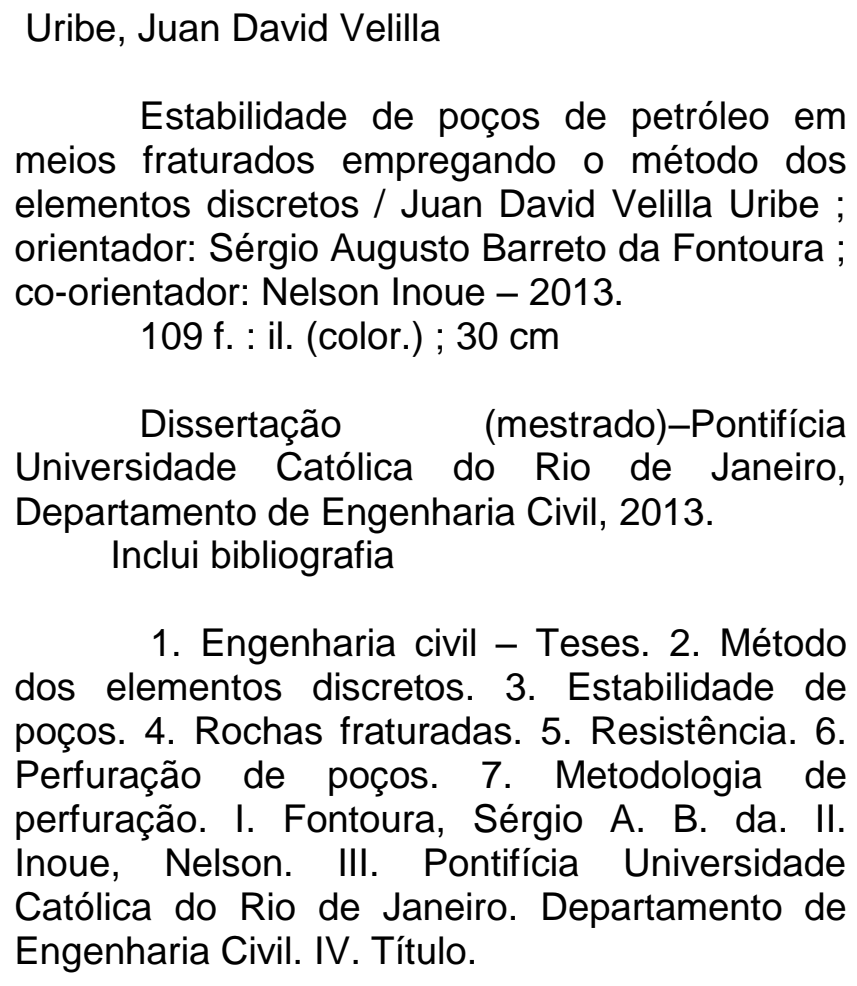

1. Engenharia civil - Teses. 2. Método dos elementos discretos. 3. Estabilidade de poços. 4. Rochas fraturadas. 5. Resistência. 6. Perfuração de poços. 7. Metodologia de perfuração. I. Fontoura, Sérgio A. B. da. II. Inoue, Nelson. III. Pontifícia Universidade Católica do Rio de Janeiro. Departamento de Engenharia Civil. IV. Título. 
Dedico esta dissertação á minha avó Elena Uribe (descanse em Paz). 


\section{Agradecimentos}

Agradeço a Deus pela fortaleza e sabedoria fornecida para terminar com sucesso esta etapa da minha vida.

Agradeço a minha avó, Helena Uribe, quem me ensinou o valor da vida e que infelizmente partiu para o céu no ano 2011. Muitas saudades de ti "Nona querida".

Agradeço a toda minha família especialmente a minha mãe, Gladys Uribe, a minha tia, Yolanda Uribe, a minha namorada, Margarita Habran, a meus irmãos, pessoas que sempre tem palavras de animo e fortaleza cada vez que eu preciso.

Agradeço ao professor Sergio A.B. da Fontoura, pela ajuda intelectual, e pela confiança depositada no meu trabalho. Sem sua ajuda não teria sido possível. Muito obrigado professor.

Agradeço ao Grupo de Tecnologia e Engenharia do Petróleo (GTEP), porque me deu a oportunidade de crescer academicamente a cada dia. Agradeço especialmente a: Nelson Inoue, Sergio Orozco, Guilherme Righeto, Carlos Emmanuel R. Lautenschläger, Pamela Rodriguez, Paola Rosas, Darwin Mateus Tarazona e Bianca F. Lima.

Agradeço ao grupo de estabilidade de poço "Wellbore Stability" da universidade industrial de Santander na Colômbia, porque graças a ele meus interesses na geomecânica do petróleo começaram a se de desenvolver.

Agradeço a meus amigos Júlio Rueda, Giovanny Rey, Mario Bonilla, Sergio Orozco, Darwin Mateus, Ruby Hernandez, Alexandre Brandão, pelos muitos momentos de estudo e alegria compartilhados, no Rio de Janeiro.

A CAPES/PROEX e à PUC-Rio, pelos auxílios concedidos, sem os quais este trabalho não poderia ter sido realizado.

Para finalizar, eu gostaria de citar algumas frases famosas de pessoas que admiro e que me motivam a ser cada dia melhor. Na vida sempre temos um 
objetivo e é a felicidade, lutar pelo que queremos nos faz grandes pessoas:

"Procure ser uma pessoa de valor, em vez de procurar ser uma pessoa de sucesso. O sucesso é consequência". (Albert Einstein, Físico).

"Algumas pessoas querem que algo aconteça, outras desejam que aconteça, outras fazem acontecer" (Michael Jordan, jogador de basquete).

"Vejo na luta enxadrística um modelo exato da vida humana, com sua luta diária, suas crises e seus incessantes altos e baixos". (Garry Kasparov, xadrezista). 


\section{Resumo}

Uribe, Juan David Velilla; Fontoura, Sérgio Augusto Barreto da (Orientador); Inoue, Nelson (Co-Orientador). Estabilidade de Poços de Petróleo em Meios Fraturados Empregando o Método dos Elementos Discretos. Rio de Janeiro, 2013. 109p. Dissertação de Mestrado Departamento de Engenharia Civil, Pontifícia Universidade Católica do Rio de Janeiro.

A estabilidade de poços de petróleo é convencionalmente analisada empregando soluções analíticas que não são adequadas para modelagem de meios fraturados, devido a suposições de meio continuo. Esta dissertação tem como objetivo principal desenvolver uma metodologia computacional para geração de janela operacional utilizando uma solução numérica, adequada para meios fraturados. No trabalho foi escolhido o software UDEC (Universal Distinct Element Code), que é baseado no método dos elementos discretos (MED). Este método considera o maciço rochoso como a união de blocos de rocha intactos, unidos pelas fraturas e cujo comportamento físico para cada elemento pode ser analisado individualmente. A modelagem computacional no UDEC foi realizada mediante uma analise hidromecânica acoplada. Esta modelagem permitiu avaliar a influencia de alguns mecanismos que governam a estabilidade de poços, como: as tensões in situ, a poropressão e a orientação, espaçamento e persistência das famílias de fraturas. Os resultados numéricos mostram o efeito das fraturas na orientação e magnitude das tensões, além da magnitude da poropressão resultando em cálculos dos limites de colapso inferior e fratura superior da rocha mais realistas.

\section{Palavras-chave}

Método dos elementos Discretos; estabilidade de poços; Rochas Fraturadas; resistência; perfuração de poços; metodologia de perfuração. 


\section{Abstract}

Uribe, Juan David Velilla; Fontoura, Sérgio Augusto Barreto da (Advisor); Inoue, Nelson (Co-Advisor). Oil Wells Stability in Fractured Media Using the Discrete Element Method. Rio de Janeiro, 2013. 109p. M.Sc. Dissertation - Departamento de Engenharia Civil, Pontifícia Universidade Católica do Rio de Janeiro.

The stability of oil wells is conventionally analyzed using analytical solutions that are often not suitable for modeling fractured media due to assumptions of continuous medium. This work has as main objective to develop a computational method for generating mud window using a numerical solution, suitable for fractured media. The software chosen for this work was the UDEC (Universal Distinct Element Code), which is based on discrete element method (DEM). This method considers the rock mass as the union of blocks of intact rock jointed by fractures, and whose physical behavior for each element can be analyzed individually. Computational modeling in UDEC was carried out in a coupled hydromechanical analysis. This modeling allowed to evaluate the influences of some of the mechanisms that govern the stability of wells, as in situ stresses, pore pressure and orientation, spacing and persistence of families of fractures. Numerical results show the effect of fracture orientation and magnitude of the stresses, besides the magnitude of the pore pressure resulting in more realistic calculations of lower collapse and upper fracture of the rock mass.

\section{Keywords}

Discrete element Method; Wellbore Stability; fractured Rocks; strength; well drilling; drilling methodology. 


\section{Sumário}

1 Introdução 20

1.1. Definição do Problema 20

1.2. Motivação 22

1.3. Objetivos 22

1.4. Estrutura do Texto 23

2 Revisão Bibliográfica $\quad 24$

2.1. Modelagem Analítica Convencional da Janela Operacional de Poços em Formações Intactas e Fraturadas 24

$\begin{array}{ll}\text { 2.1.1. Modelagem analítica de descontinuidades } & 27\end{array}$

2.2. Panorama da Modelagem Analítica de Estabilidade de Poços em Formações $\begin{array}{ll}\text { Fraturadas } & 28\end{array}$

2.3. Panorama da Modelagem Numérica de Estabilidade de Poços em

Formações Fraturadas 31

3 O Método dos Elementos Discretos (MED) para Rochas Fraturadas 45

3.1. Que é o MED? 45

3.2. Formulação do Método dos Elementos Discretos (MED) 46

3.2.1. Considerações Físicas 48

- Movimentação dos Blocos de Rocha 48

- Equilíbrio de Momento e Energia 50

- Deformabilidade dos Blocos de Rocha 51

- Deformabilidade das Fraturas 53

- Fluxo de fluido nas fraturas 56

3.2.2. Considerações numéricas $\quad 59$

- Representação Numérica das Descontinuidades 59

- Discretização Nodal Mista para Deformação em uma Rede Triangular 61

- Discretização Nodal Mista para Tensões em uma Rede Triangular 62

- Condições de contorno 64 
- Determinação do Passo de Tempo Mecânico: Solução Estável

3.3. O software UDEC (Universal Distinct Element Code) 66

- Etapa 1: Definição da Geometria do Problema 67

- Etapa 2: Discretização por Diferenças Finitas 67

- Etapa 3: Modelos Constitutivos e Propriedades dos Materiais 67

- Etapa 4: Condições de Contorno 67

- Etapa 5: Utilidades 67

- Etapa 6: Configurações 68

- Etapa 7: Execução do problema 68

- Etapa 8: Gráficas 68

4. Modelagem computacional da estabilidade de poços em rochas fraturadas 70

4.1. Etapa 1: Consolidação inicial do maciço rochoso 71

4.1.1. Geometrias propostas 71

4.1.2. Modelos constitutivos e tensões in situ 72

4.1.3. Condições de contorno e variáveis numéricas 74

4.1.4. Condição final de equilíbrio 76

4.2. Etapa 2: Escavação do furo e determinação das pressões de colapso 76

4.2.1. Escavação e aplicação da pressão do fluido em torno do furo 77

4.2.2. Determinação das pressões de colapso superior e inferior 79

4.3. Resultados e discussão 79

4.3.1. Parede do poço impermeável 79

4.3.2. Parede do poço permeável 84

4.3.3. Efeito da orientação das famílias de fraturas na estabilidade de poços 87

5. Validação e comparação dos resultados analíticos e numéricos 90

5.1. Janela operacional analítica versus numérica 90

5.2. Distribuição de tensões em torno e longe da face do poço 94

5.3. Distribuição de poropressão na modelagem analítica Vs Numérica 98

6 Conclusões e Sugestões 99

6.1. Conclusões 99

6.2. Sugestões para trabalhos futuros 100 
Apêndice A 


\section{Lista de Figuras}

Figura 1. 1- Os quatro mecanismos mais importantes de instabilidade de poço (Pasic, 2007). .21

Figura 1. 2- Perfuração através de formações fraturadas (Pasic, 2007). 22

Figura 2. 1 - llustração dos tipos de ruptura na parede do Poço, a) por Tração e

b) por Cisalhamento (Modificado, Fjaer, 2008). 25

Figura 2. 2- Efeito do incremento do peso da lama na parede do poço (Pasic, 2007) 25

Figura 2. 3 - Modelo de estabilidade mecânico analítico da rocha em torno do poço. 26

Figura 2. 4 - Parâmetros de perfuração durante as três tentativas de perfuração em um poço de petróleo em camadas fraturadas vulcânicas (Santarelli et al, 1992)

Figura 2. 5 - Comportamentos das fraturas no modelamento, a) Aberturas das fraturas para uma densidade da lama de $1.2 \mathrm{~g} / \mathrm{cm}^{3}$, b) Aberturas das fraturas para uma densidade da lama de $1.7 \mathrm{~g} / \mathrm{cm}^{3}$, c) Deslocamento cisalhante ao longo das farturas para um mergulho de $45^{\circ}$ e $1.7 \mathrm{~g} / \mathrm{cm}^{3}$. (Santarelli et al, 1992). .33

Figura 2. 6 - Fluxo de fluido nas fraturas, a) Taxa de fluxo através das fraturas com mergulho de $45^{\circ}$ e densidade do fluido de $1.2 \mathrm{~g} / \mathrm{cm}^{3}$ e $1.3 \mathrm{~g} / \mathrm{cm}^{3}$, b) Fraturas abertas quando o mergulho é $20^{\circ}$ e a densidade $1.2 \mathrm{~g} / \mathrm{cm}^{3}$. (Santarelli, 1992).

Figura 2. 7 - Contornos de cisalhamento depois da escavação para a geometria circular e uma pressão da lama de $10 \mathrm{MPa}$, a) $\sigma_{\mathrm{h}}=45 \mathrm{MPa}, \sigma_{\mathrm{H}}=45 \mathrm{MPa}, \mathrm{b}$ ) $\sigma_{\mathrm{h}}=45 \mathrm{MPa}, \sigma_{\mathrm{H}}=67.5 \mathrm{MPa}, \mathrm{c}$ ) $\sigma_{\mathrm{h}}=45 \mathrm{MPa}, \sigma_{\mathrm{H}}=90 \mathrm{MPa}$, (Zhang et al ,1999) 35

Figura 2. 8 - Comparação dos resultados do modelamento analítico e numérico para $\sigma_{\mathrm{h}}=45 \mathrm{MPa}, \sigma_{\mathrm{H}}=67.5 \mathrm{MPa}$ (Zhang et al ,1999). 36

Figura 2. 9 - Taxas de fluxo através das fraturas para uma pressão da lama de $10 \mathrm{MPa}$ a) $\sigma_{\mathrm{h}}=45 \mathrm{MPa}, \sigma_{\mathrm{H}}=45 \mathrm{MPa}$; a máxima taxa de fluxo é $0.04 \mathrm{~m}^{2} / \mathrm{s} \mathrm{b}$ ) $\sigma_{\mathrm{h}}=45 \mathrm{MPa}, \sigma_{\mathrm{H}}=67.5 \mathrm{MPa}$; a máxima taxa de fluxo é $0.033 \mathrm{~m}^{2} / \mathrm{s} \mathrm{c}$ ) $\sigma_{\mathrm{h}}=45$ $\mathrm{MPa}, \sigma_{\mathrm{H}}=90 \mathrm{MPa}$; a máxima taxa de fluxo é $0.032 \mathrm{~m}^{2} / \mathrm{s}$ (Zhang et al ,1999) 
Figura 2. 10 - Resultados do modelamento para o caso 2, a) parede impermeável com vetores de deslocamento e mudanças na poropressão (linha espessa), b) parede permeável com vetores de deslocamento e mudanças na poropressão (linha espessa), c) parede permeável com ângulo de atrito constante e com as fraturas no limite de equilíbrio e d) parede permeável com redução do ângulo de atrito e com as fraturas no limite de equilíbrio (Chen et al, 2003). .38

Figura 2. 11 - Máxima tensão e deslocamento de cisalhamento ao redor do poço versus mergulho da família de fraturas (Yamamoto et al, 2002)........39

Figura 2. 12 - Máximo deslocamento de cisalhamento ao redor do poço versus

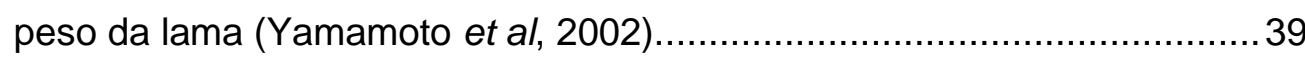

Figura 2. 13 - a) deslocamentos na direção $x$, b) deslocamentos na direção y, c) zonas colapsadas no poço (zonas em azul) (Nicolson \& Hunt, 2004).

Figura 2. 14 - Predições analíticas de dano ao redor do poço, a) modelo homogêneo isotrópico, b) modelo transversalmente isotrópico (Willson et al, 2007)

Figura 2. 15 - Predições numéricas de estabilidade de poço utilizando o modelo de elementos discretos (Willson et al, 2007).

Figura 2. 16 - Comportamentos da rocha durante o carregamento biaxial, para mergulhos de a) $30^{\circ}$ (foto ampliada), a) $30^{\circ}$ (detalhe), a) $45^{\circ}$, a) $60^{\circ}$ (Sagong et al,2011). 44

Figura 2. 17 - Orientação e magnitude dos deslocamentos das esferas ao redor do furo para um orientação de a) $30^{\circ}$, b) $45^{\circ}$ e c) $60^{\circ}$ (Sagong et al ,2011). 44

Figura 3.1 - Perfuração de um poço em um maciço rochoso fraturado (manual UDEC, 2011) 46

Figura 3. 2- Fluxograma das principais considerações físicas e numéricas envolvidas no MED.

Figura 3. 3 - Natureza entrelaçada do ciclo de calculo utilizado na formulação dos elementos discretos (manual UDEC, 2011). 49

Figura 3.4 - Zoneamento dentro do modelo contendo um sistema de descontinuidades continua e descontinua (manual UDEC, 2011). 52

Figura 3.5 - Modelo de escorregamento de Coulomb para o comportamento básico da fratura (Zhang et al, 1999)....................................................55

Figura 3.6 - Comportamento da deformação hidráulico- mecânica no MED, a) pressão do fluido causando efeitos mecânicos; b) deformação do bloco 
afetando a apertura hidráulica a; c) fluxo de fluidos afetado pela apertura a,

d) Geração de pressão diferencial do fluido (Zhang et al, 1999). 56

Figura 3.7 - Fluxo nas fraturas modeladas como fluxo entre domínios hidráulicos

(Zhang et al, 1999).

Figura 3.8 - Relação entre apertura hidráulica, a e tensão normal na fratura, $\sigma_{n}$

(Zhang et al, 1999) 59

Figura 3.9 - Contato entre dois blocos rígidos (manual UDEC, 2011). 60

Figura 3.10 - Definição dos contatos no MED, a) contato limite de esquina arredondado, b) interação esquina-esquina (manual UDEC, 2011) 60

Figura 3.11 - Contatos e domínios entre dois blocos deformáveis (manual

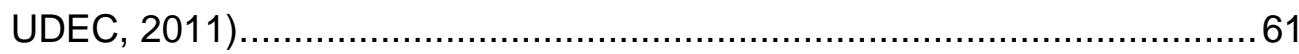

Figura 3. 12 - O software UDEC (Itasca 2011) ..........................................66

Figura 3. 13 - Janela principal do software UDEC (Itasca, 2011) .....................68

Figura 4. 1- Procedimento geral de cálculo na modelagem computacional. ......70 Figura 4. 2 - Geometria global para os modelos propostos. .......................... 71 Figura 4. 3 - Geometrias analisadas, a) modelo homogêneo isotrópico, b) modelo transversalmente isotrópico, c) modelo anisotrópico. ...................72

Figura 4. 4 - Condições de contorno aplicadas para os casos 10 e $12 \ldots \ldots \ldots \ldots . . .74$

Figura 4.5 - Poropressão atuando nas fraturas para os casos 7 e 8 ................ 75

Figura 4. 6 - Malha de diferenças finitas para os casos 9, 10, 11 e 12 .............75

Figura 4.7 - História da máxima força de desequilíbrio na etapa 1 para o caso 5 . .76

Figura 4. 8 - Aplicação da sobre pressão nos contatos ao redor do furo depois da escavação para os casos 1 até 4 . .77

Figura 4. 9 - História da máxima força de desequilíbrio para o caso 9. 77

Figura 4. 10 - Poropressão no estado final de equilíbrio na parede impermeável no caso 8 e uma pressão da lama de $20 \mathrm{MPa}$. .78

Figura 4. 11 - Poropressão no estado final de equilíbrio para o caso 8 na parede permeável a uma pressão da lama de 23.5 MPa. .78

Figura 4. 12 - Funções FISH para a) obter os elementos plastificados em torno do furo e b) para obter as fraturas no limite de atrito. 79

Figura 4. 13 - Elementos plastificados para, a) baixa pressão de 23. $5 \mathrm{MPa}$ e b) alta pressão de $66 \mathrm{MPa}$.

Figura 4. 14 - Elementos plastificados para o caso 8, a) pressão de $12 \mathrm{MPa}$ e b) alta pressão de $60 \mathrm{MPa}$. 
Figura 4. 15 - Elementos plastificados para o caso 12, a) pressão de $24 \mathrm{MPa}$ e

b) pressão de $70 \mathrm{MPa}$.

Figura 4. 16 - Fraturas no limite de atrito para o caso 7, a) pressão de $12 \mathrm{MPa}$ e

b) pressão de $60 \mathrm{MPa}$ 82

Figura 4. 17 - Fraturas no limite de atrito para o caso 12, a) pressão de $24 \mathrm{MPa}$ e b) pressão de $70 \mathrm{MPa}$.

Figura 4. 18 - Direção das tensões principais no caso 7 para uma pressão da lama de $5 \mathrm{MPa}$. 83

Figura 4. 19 - Direção das tensões principais no caso 7 para uma pressão da lama de $35 \mathrm{MPa}$. 83

Figura 4. 20 - Fluxo de fluidos nas fraturas para o caso 8 com um peso de lama nas paredes do poço de $23.5 \mathrm{MPa}$. .85

Figura 4. 21 - Fluxo de fluidos nas fraturas para o caso 8 com um peso de lama nas paredes do poço de $59 \mathrm{MPa}$. .85

Figura 4. 22 - Fluxo de fluidos nas fraturas para o caso 12 com um peso de lama nas paredes do poço de $23.5 \mathrm{MPa}$ .86

Figura 4. 23 - Fluxo de fluidos nas fraturas para o caso $12 \mathrm{com}$ um peso de lama nas paredes do poço de $59 \mathrm{MPa}$. 86

Figura 4. 24 - Zonas plastificadas a peso da lama de $23.5 \mathrm{MPa}$ e para um mergulho de fraturas de a) $45^{\circ}$, b) $20^{\circ}$ e c) $70^{\circ}$. 87

Figura 4. 25 - Fraturas no limite de atrito para um peso da lama de $23.52 \mathrm{MPa}$ e para um mergulho de fraturas de a) $45^{\circ}$, b) $20^{\circ}$ e c) $70^{\circ}$. 88

Figura 4. 26 - Zonas plastificadas a peso da lama de $59 \mathrm{MPa}$ e para um mergulho de fraturas de a) $45^{\circ}$, b) $20^{\circ}$ e c) $70^{\circ}$ 88

Figura 4. 27 - Fraturas no limite de atrito para um peso da lama de $59 \mathrm{MPa}$ e para um mergulho de fraturas de a) $45^{\circ}$, b) $20^{\circ}$ e c) $70^{\circ}$. 89

Figura 5. 1 - Comparação dos elementos plastificados utilizando o UDEC e o SEST para o caso 4 e uma pressão de a) $23.5 \mathrm{MPa}$ e b) $63.61 \mathrm{MPa}$.........92 Figura 5. 2 - Comparação dos elementos plastificados utilizando o UDEC e o SEST para o caso 7 e uma pressão de a) $11.76 \mathrm{MPa}$ e b) $59 \mathrm{MPa}$. 93

Figura 5. 3 - Comparação dos elementos plastificados utilizando o UDEC e o SEST para o caso 12 e uma pressão de a) $23.5 \mathrm{MPa}$ e b) $70.57 \mathrm{MPa}$.......93

Figura 5. 4 - Tensões principais analíticas atuando ao redor de um poço vertical para baixas pressões de fluido de perfuração. .94

Figura 5. 5 - Tensões principais analíticas e numéricas atuando ao redor do poço para o caso 4 e uma pressão de $37.6 \mathrm{MPa}$. .95 
Figura 5. 6 - Tensões analíticas e numéricas atuando distante da parede do poço na direção da tensão horizontal mínima para o caso 4 e uma pressão

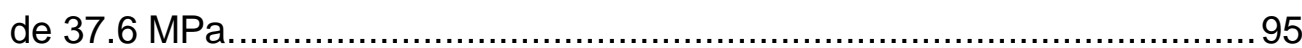

Figura 5.7 - Tensões analíticas e numéricas atuando distante da parede do poço na direção da tensão horizontal máxima para o caso 4 e uma pressão de 37.6 MPa .96

Figura 5. 8 - Tensões principais analíticas e numéricas atuando ao redor do poço para o caso 12 e uma pressão de $23.5 \mathrm{MPa}$. .96

Figura 5. 9 - Tensões analíticas e numéricas atuando distante da parede do poço na direção da tensão horizontal mínima para o caso 12 e uma pressão de $23.5 \mathrm{MPa}$

Figura 5. 10 - Tensões analíticas e numéricas atuando distante da parede do poço na direção da tensão horizontal máxima para o caso 12 e uma pressão de $23.5 \mathrm{MPa}$.

Figura 5. 11 - Distribuição da poropressão longe da parede do poço impermeável, para o modelo analítico e numérico. .98

Figura A. 1 - Transformação de tensões num sistema coordenado 108

Figura A. 2 - Tensões em coordenadas cilíndricas atuando ao redor do poço. 108 


\section{Lista de Tabelas}

Tabela 1. 1 - Causas de Instabilidade de Poço (McLellan et al, 1994). 20

Tabela 4.1 - Propriedades geométricas dos modelos propostos. ....................72

Tabela 4. 2 - Propriedades da rocha intacta e fratura....................................... 73

Tabela 4.3 - Tensões in situ para os 12 casos propostos............................... 74

Tabela 4. 4 - Janela operacional para os casos propostos na parede do poço

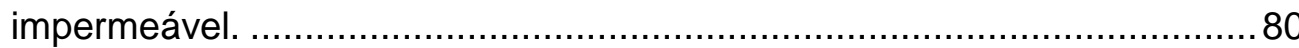

Tabela 4. 5 - Janela operacional para os casos propostos na parede do poço permeável. .84

Tabela 5. 1 - comparação da Janela operacional analítica versus numérica para os casos propostos na parede do poço impermeável. 91

Tabela 5. 2 - comparação da Janela operacional analítica versus numérica para os casos propostos na parede do poço permeável. 


\section{Lista Símbolos}

a Abertura hidráulica.

a Coeficiente de amortecimento.

C Coesão.

$\varepsilon \quad$ Deformação.

$\mathrm{F} \quad$ Força.

$g \quad$ Aceleração da gravidade.

$\dot{\theta} \quad$ Velocidade angular.

I Inercia.

$\lambda \quad$ Constante de Lamé.

$\zeta \quad$ Parâmetro de Kronecker.

K Rigidez.

$K_{j} \quad$ Fator de permeabilidade.

$l \quad$ Comprimento do contato.

$m$ Massa.

$u \quad$ Deslocamento.

$\dot{u} \quad$ Velocidade.

$\ddot{u} \quad$ Aceleração.

t Tempo.

$P_{p}$ poropressão.

Q Vasão.

$\sigma^{\prime} \quad$ Tensão efetiva.

$\sigma_{1} \quad$ Tensão principal maior.

$\sigma_{2} \quad$ Tensão principal intermedia.

$\sigma_{3} \quad$ Tensão principal menor.

$\sigma_{\theta} \quad$ Tensão tangencial.

$\sigma_{z} \quad$ Tensão axial.

$\sigma_{r} \quad$ Tensão radial.

$\sigma_{H} \quad$ Tensão Horizontal maior.

$\sigma_{h} \quad$ Tensão Horizontal menor.

$\sigma_{n} \quad$ Tensão normal.

$\sigma_{V} \quad$ Tensão Vertical.

$\sigma_{x} \quad$ Tensão normal na direção do eixo $\mathrm{X}$. 


$$
\begin{array}{ll}
\sigma_{y} & \text { Tensão normal na direção do eixo } \mathrm{Y} . \\
\sigma_{z} & \text { Tensão normal na direção do eixo } \mathrm{Z} . \\
\tau_{s} & \text { Tensão de cisalhamento. } \\
\tau_{x y} & \text { Tensão de cisalhamento no plano xy. } \\
\tau_{x z} & \text { Tensão de cisalhamento no plano xz. } \\
\tau_{y z} & \text { Tensão de cisalhamento no plano yz. } \\
\Phi & \text { Ângulo de atrito. } \\
V & \text { Volume. }
\end{array}
$$

